\title{
PERCEPCIONES, ACTITUDES Y CONDUCTAS DE MÉDICOS GINECO- OBSTETRAS, RESPECTO AL USO ACTUAL DE TERAPIA DE REEMPLAZO HORMONAL
}

\author{
Ernesto Pizarro O., Mauricio Villarroel C., Oscar Fertilio T., Ana Gadán H., Marcelo \\ González V., Agustín Ibarra P., Manuel Parra A., Rodrigo Riveros K., César Sandoval \\ S., Patricio Silva R., Víctor Valverde $P$.
}

Unidad de Climaterio, Menopausia y Endocrinología Ginecológica, Departamento de Obstetricia y Ginecología, Clínica Alemana.

\section{RESUMEN}

Resultados de una encuesta con preguntas específicas contestadas por el $97,6 \%$ de los ginecoobstetras del Departamento de Obstetricia y Ginecología de Clínica Alemana de Santiago, para establecer un diagnóstico de situación sobre el uso de terapia de reemplazo hormonal (TRH). Se enfatizan preguntas sobre la aceptabilidad de las futuras usuarias tanto de TRH como de terapias alternativas; sobre la percepción de los eventuales beneficios desde un punto de vista general, o específicamente cardiovascular; así como las preferencias de hormonas a usar y predilecciones por las vías de administración; las asociaciones del uso y cáncer mamario; las variaciones sobre el uso de TRH pos publicación del Women Health Iniciative Study; los factores que inciden en la discontinuación del tratamiento; la importancia de edad para el uso, factores relacionados con los costos de las terapias y la preferencia o no de productos originales de investigación. Conclusión: Es necesaria una mayor capacitación sobre el uso de TRH en la actualidad tanto de los ginecólogos especialistas en climaterio y menopausia, como de los que no lo son. A falta de información concluyente sobre esta temática en el momento actual se sugiere que el manejo de este tipo de pacientes sea efectuado por los equipos médicos especializados.

\section{PALABRAS CLAVE: Menopausia, climaterio, terapia de reemplazo hormonal}

\section{SUMMARY}

In order to set a diagnosis about the use of hormonal replacement therapy (HRT) in peri and postmenopausal women by the obstetricians and gynecologists members of our staff, we did a survey that was answered by $97.6 \%$ of them. Questions about the future patients desire related to use of HRT or use of natural or alternative medicines were included. There were also questions related to the "risk-benefit" relationship on the use of HRT from an overall point of view as well as from a cardiovascular point of view. The preference of medical doctors (MD) on choice of hormone type in HRT use, in respect to the administration, were also asked. Among others, questions were asked in order to know MD opinions on the relationship with breast cancer, the changes on acceptability use after the WHI study, reasons for the treatment discontinuation, the importance of women's age and the cost of pharmaceuticals products on HRT use. Conclusions: Are focused on a need of a solid educational program on use of HRT addressed to gynecologists, as well as to general practitioners, cardiologists, oncologists and others related. Educational programs are also quite beneficial to future users as well as women that are currently under treatment. In the absence of conclusive information available at the present moment, we advise that patients considering HRT should seek the counsel of medical teams whose specialties include menopause.

KEY WORDS: Menopause, climacteric, hormonal replacement therapy 


\section{INTRODUCCIÓN}

Los resultados del Women Health Iniciative Study (WHI) comunicados a mediados del año 2002 impactaron fuertemente a la comunidad ginecoobstétrica mundial. Hasta ese momento los estudios observacionales existentes apuntaban a apoyar las bondades atribuibles a la terapia de reemplazo hormonal (TRH) en la peri y postmenopausia en el corto y mediano plazo y, eventualmente en el largo plazo. Entre otras conclusiones, el estudio WHI ponía una voz de alarma y duda sobre algunos de los reales beneficios atribuidos hasta ese momento a la TRH, como lo eran aquellos sobre el sistema cardiovascular y el neurocognitivo. Igualmente el estudio WHI evidenciaba un mayor incremento en el riesgo de cáncer mamario después de 5 años de uso de TRH combinada continua en la postmenopausia con uso de estrógenos conjugados de equinos en dosis de $0,625 \mathrm{mg} / \mathrm{día}$ más acetato de medroxiprogesterona $2,5 \mathrm{mg} / \mathrm{día}$ (1). Algunas instituciones reguladoras del uso de medicamentos, como el Food and Drug Administration (FDA) llegaron aún a afirmar que el uso de TRH solo debería ser administrada "por el menor tiempo posible, a las dosis más bajas posibles y ser prescritas solo si la presencia de sintomatología así lo ameritaba" (2). Como agravante se señalaba el hecho de ser el WHI un estudio experimental, prospectivo, aleatorio, ciego, multicéntrico, de gran casuística, con largo tiempo de observación, diseñado por expertos de un país desarrollado pionero en la investigación mundial, como lo son los EEUU de Norteamérica, y que además cumplía a cabalidad los conceptos requeridos por la denominada "medicina basada en la evidencia" (3). Como contraparte, el estudio WHI señalaba bondades, en mujeres histerectomizadas, como menor riesgo sobre cáncer de colon, osteoporosis, y paradojalmente, menor riesgo de cáncer mamario (no estadísticamente significativo), después del uso continuo de estrógenos conjugados de equino solos en dosis de 0,625 mg/día (4).

Análisis posteriores derivadas del mismo estudio WHI (5-9) y de otros (10-14) han llevado a sugerir que si el uso de TRH es iniciado precozmente después de la postmenopausia predominarán en el largo plazo los efectos protectores sobre el aparato cardiovascular.

Las percepciones y actitudes médicas ante el uso o no, de una determinada terapia es un hecho subjetivo, muy personal, que puede estar influenciado por diversos factores como lo son, entre otros, la información disponible a la fecha sobre esa terapia, el acceso y la capacidad o interés de asimilación de cada médico de dicha información, la experiencia personal del médico con el uso previo de dichas terapias, el deseo de uso, o aceptabilidad, por parte de las pacientes así como la información previa de las mismas, y la positiva, negativa o alarmista postura de los medios de comunicación al respecto. Además, la creciente magnitud de la información disponible, con a veces acentuadas divergencias sobre la misma, así como la alta sobreespecialización médica existente, que es de especial relevancia en los centros de alta especialización como el nuestro, son factores, entre otros, que atentan contra la uniformidad de criterios médicos con las consecuentes divergentes percepciones y actitudes sobre el uso de una específica terapia médica.

Con el objetivo de establecer un diagnóstico de situación respecto a la uniformidad o discordancia de la opinión médica sobre diversos factores específicos que inciden en el uso de la TRH en el momento actual, es que se diseñó una encuesta a ser respondida por todos los médicos ginecoobstetras del Departamento de Obstetricia y Ginecología de la Clínica Alemana de Santiago.

\section{MATERIAL Y MÉTODO}

El Departamento de Obstetricia y Ginecología de la Clínica Alemana de Santiago contaba al mes de noviembre de 2006, con 43 ginecoobstetras divididos en distintas Unidades y Secciones de Alta Especialización. La Unidad de Climaterio y Menopausia y Endocrinología Ginecológica estaba compuesta a esa fecha por 10 ginecoobstetras. Clínica Alemana es una clínica privada que efectúa paralelamente a la función asistencial, actividades académicas universitarias de pre y post grado, así como actividades de investigación y extensión. En la labor asistencial, predomina la atención a una población de pacientes de mediano y alto nivel socioeconómico. Durante el mes de noviembre de 2006 se distribuyó la encuesta adjunta, la cual se solicitó ser respondida en forma anónima por los 41 ginecoobstetras miembros del Departamento, que se encontraban disponibles para ser encuestados en ese momento. La encuesta fue previamente validada por el grupo autor. En diciembre de 2006 se recolectaron las respuestas de 40 de las 41 encuestas distribuidas. Del total de éstas, 10 médicos se manifestaron como expertos o especialistas en climaterio y menopausia y 30 manifestaron que sus apreciaciones al respecto fueron proporcionadas desde un punto de vista de un ginecólogo general. 


\section{RESULTADOS}

Los resultados de las encuestas se presentan en las Tablas I a V. La Tabla I presenta percepciones sobre generalidades en el uso de TRH. La Tabla II hace referencia a predilecciones en los tipos y vías a usar en la TRH, así como a las contraindicaciones de las mismas. La Tabla III hace especial referencia al factor etario en el uso de TRH. La Tabla IV relaciona las percepciones y actitudes médicas respecto al estudio $\mathrm{WHI}$, así como a las inquietudes en caso ocurrir demandas judiciales como consecuencia de serios efectos adversos derivados del uso de TRH. La Tabla V contiene percepciones y actitudes respecto al deseo de uso de medicinas naturales o alternativas tanto por parte de los médicos como de las usuarias; en esta tabla también se incluyen preguntas sobre factores que pueden tener importancia en la discontinuación de la TRH en la práctica diaria.

\section{DISCUSIÓN}

Las encuestas constituyen una metodología rápida, simple y de bajo costo para obtener información sobre una determinada materia, en este caso específico, las percepciones y actitudes sobre el uso de la TRH por parte de todos los médicos ginecoobstetras miembros de un centro docente asistencial común, como lo es Clínica Alemana.

Este es un centro de alta complejidad que cuenta en el Departamento de Obstetricia y Ginecología con médicos altamente especializados en Unidades Específicas de atención como lo son aparte de la nuestra, las Unidades de Adolescencia, Medicina Reproductiva, Perinatología, Oncología Ginecológica, Uroginecologia y Ecografía Ginecoobstetrica.

Si bien la población encuestada que responde a la encuesta es pequeña ( $n: 40)$ corresponde a la casi totalidad de los miembros del Departamento $(97,6 \%)$ por lo que ella refleja fidedignamente el sentir de los componentes que trabajan en este centro docente asistencial.

Resalta notoriamente al analizar las respuestas obtenidas, ante una pregunta específica, la diversidad de las mismas. Esto puede ser fácilmente entendible debido a los factores subjetivos personales en las percepciones y actitudes médicas las cuales son totalmente individuales y que ya fueron enunciadas en la introducción de esta presentación. Se agrega a este hecho que el estudio WHI, que si bien fue comunicado hace 5 años, aún genera en estos momentos nuevas informaciones en subgrupos poblacionales del mismo, las cuales han en- tregado informaciones aclaratorias muchas veces encontradas o contradictorias con las reportadas inicialmente con las consecuentes confusiones en las futuras usuarias y en la población médica misma. Por último, se agrega además, el ya señalado hecho de ser nuestra Clínica un centro médico de alta especialización, en la cual existen profesionales que priorizan otras áreas de la ginecoobstetricia, ajenas al climaterio y la menopausia.

Al analizar algunos de los resultados obtenidos destaca el apreciar que la mayoría de los médicos $(67,5 \%)$, percibe "desconfianza o temor" por parte de las pacientes al futuro uso de TRH versus solo el $27,5 \%$ que percibe en las candidatas "confianza u optimismo" al eventual futuro uso de TRH. Este punto nos parece como de relevante importancia en la indicación de TRH misma, en que un médico "inseguro de los reales beneficios" de la TRH $(32,5 \%)$, "dubitativo" de la magnitud real de los riesgos del uso de la $\mathrm{TRH}(45 \%)$, y que siente que existe una influencia negativa de la prensa al uso de TRH (70\%), o una influencia negativa por parte de médicos de otras especialidades (cardiólogos, oncólogos) (75\%), o de amistades o parientes de la paciente $(72,5 \%)$, puede decidir por no tener una conducta positiva o actitud convincente en la indicación de TRH y tienda a adecuarse plenamente al concepto de "decisión conjunta informada" respetando desde los primeros momentos el negativo deseo al uso de TRH por parte de la paciente. Más aún, cuando el $37,5 \%$ de los ginecólogos manifiesta una mediana $o$ alta inquietud a ser demandado en caso ocurrir un evento serio adverso con la TRH por él prescrita.

La percepción de una disminución al futuro uso de TRH por parte de las pacientes se ha visto reflejada también en otros países, como fue la disminución de las ventas (38\%) en países como los EEUU de Norteamérica en el año siguiente a la publicación inicial del estudio WHI (15) o la disminución de las ventas que se produjo en nuestro país en los años siguientes a la publicación de dicho estudio (16) (2.315.667 de cajas de TRH vendidas en el 2002 versus 1.074 .639 vendidas en 2006) o la baja en las ventas de la TRH total, en los países donde se comercializa la tibolona original de marca (no incluye USA), en que las ventas anuales de 40.000 .000 de euros en el 2001 cayeron a 20.000 .000 de euros en el 2005 (17).

Además, en esta publicación se evalúan, entre otras, las percepciones de los médicos ginecólogos sobre el uso de la TRH desde el punto de vista general $(57 \%$ estiman más beneficios que riesgos al usar al TRH), cardiovascular ( $45 \%$ estiman más 


\section{Tabla I}

\section{PREGUNTAS RELATIVAS A GENERALIDADES DEL USO DE TRH}

\begin{tabular}{|c|c|c|c|}
\hline $\begin{array}{l}\text { PREGUNTA } \\
\text { 1. Su inquietud sobre climaterio y } \\
\text { menopausia es la de un....... }\end{array}$ & $\begin{array}{l}\text { ALTERNATIVAS } \\
\text { a) especialista en climaterio y } \\
\text { menopausia } \\
\text { b) ginecólogo general }\end{array}$ & $\begin{array}{l}\text { RESPUESTAS } \\
\qquad \begin{array}{r}10 \\
30\end{array}\end{array}$ & $\begin{array}{l}\% \\
25 \\
75\end{array}$ \\
\hline $\begin{array}{l}\text { 2. Estima usted que la TRH, en } \\
\text { general, tiene para las pacientes....... }\end{array}$ & $\begin{array}{l}\text { a) más beneficios que riesgos } \\
\text { b) semejantes riesgos y beneficios }\end{array}$ & $\begin{array}{l}23 \\
13\end{array}$ & $\begin{array}{l}57,5 \\
32,5\end{array}$ \\
\hline $\begin{array}{l}3 . . . . y \text { desde el punto de vista } \\
\text { cardiovascular.... }\end{array}$ & $\begin{array}{l}\text { a) más riesgos que beneficios. } \\
\text { b) más beneficios que riesgos } \\
\text { c) semejantes riesgos y beneficios } \\
\text { d) sin respuesta }\end{array}$ & $\begin{array}{r}18 \\
14 \\
7 \\
1\end{array}$ & $\begin{array}{r}45 \\
35 \\
17,5 \\
2,5\end{array}$ \\
\hline $\begin{array}{l}\text { 4. Según usted, en general la } \\
\text { percepción inicial por parte de las } \\
\text { candidatas al uso de TRH por } \\
\text { primera vez, es............ }\end{array}$ & $\begin{array}{l}\text { a) desconfianza o temor al futuro } \\
\text { uso } \\
\text { b) confianza u optimismo con el } \\
\text { futuro uso } \\
\text { c) sin respuesta }\end{array}$ & $\begin{array}{r}27 \\
11 \\
2\end{array}$ & $\begin{array}{c}67,5 \\
27,5 \\
5\end{array}$ \\
\hline $\begin{array}{l}\text { 5. ¿Indica habitualmente, en este } \\
\text { grupo de pacientes, fármacos } \\
\text { para el tratamiento de las } \\
\text { siguientes patologías? }\end{array}$ & & & \\
\hline a) Hipertensión arterial & $\begin{array}{l}\text { si } \\
\text { no }\end{array}$ & $\begin{array}{r}6 \\
34\end{array}$ & $\begin{array}{l}15 \\
85\end{array}$ \\
\hline b) Hipercolesterolemia & $\begin{array}{l}\text { si } \\
\text { no } \\
\text { sin respuesta }\end{array}$ & $\begin{array}{r}18 \\
21 \\
1\end{array}$ & $\begin{array}{r}45 \\
52,5 \\
2,5\end{array}$ \\
\hline c) Osteopenia/osteoporosis & $\begin{array}{l}\text { si } \\
\text { no }\end{array}$ & $\begin{array}{r}35 \\
5\end{array}$ & $\begin{array}{l}87,5 \\
12,5\end{array}$ \\
\hline $\begin{array}{l}\text { 6. ¿ Tiene en cuenta el costo del } \\
\text { medicamento cuando indica } \\
\text { TRH? }\end{array}$ & $\begin{array}{l}\text { si } \\
\text { no } \\
\text { sin respuesta }\end{array}$ & $\begin{array}{r}21 \\
18 \\
1\end{array}$ & $\begin{array}{l}52,5 \\
45 \\
2,5\end{array}$ \\
\hline $\begin{array}{l}\text { 7. ¿Tiene preferencias en recetar } \\
\text { productos farmacológicos } \\
\text { originales de investigación versus } \\
\text { productos similares sin respaldo } \\
\text { de investigación? }\end{array}$ & $\begin{array}{l}\text { si, a precio similar } \\
\text { si, aunque sean más costosos } \\
\text { no }\end{array}$ & $\begin{array}{r}13 \\
25 \\
2\end{array}$ & $\begin{array}{c}32,5 \\
62,5 \\
5\end{array}$ \\
\hline
\end{tabular}

riesgos que beneficios) y solo un 35\%, estiman más beneficios que riesgos al usar $\mathrm{TRH}$; la importancia de la edad de la mujer para la indicación de $\mathrm{TRH}$ $(92,5 \%$, estima que es importante), si existen limites superiores de edad para indicar su uso (opciones SI 52,5\% y NO 45\%), si hay limites en el tiempo máximo de uso de TRH (opción SI, 70\%), si existe una asociación significativa con riesgo de cáncer de mama al usar TODO tipo de TRH (opción SI, solo un $10 \%$ ), o si ésta asociación se produce al usar SOLO algunos tipos y dosis hormonales (opción $\mathrm{SI}, 87,5 \%$ ), así como se evalúa también a 4,5 años de la publicación del estudio WHI si los datos por él aportados parecen ser altamente fiables (solo el $12,5 \%$ de los encuestados ), o medianamente fiables $(60 \%)$ o no fiables $(5 \%)$, agregándose un $22,5 \%$ (9 casos) en que los profesionales manifiestan estar incapacitados para opinar.

El $80 \%$ de los médicos encuestados siente que después del estudio WHI su actitud ha cambiado respecto al uso de $\mathrm{TRH}$. Digno de atención es el apreciar que el $77,5 \%$ de los médicos estima que es alta o mediana "la confianza y el deseo" por parte de las pacientes por el uso de medicinas alter- 
Tabla II

PREGUNTAS RELACIONADAS CON PREDILECCIONES DE TIPOS Y VÍAS DE HORMONAS A USAR EN TRH

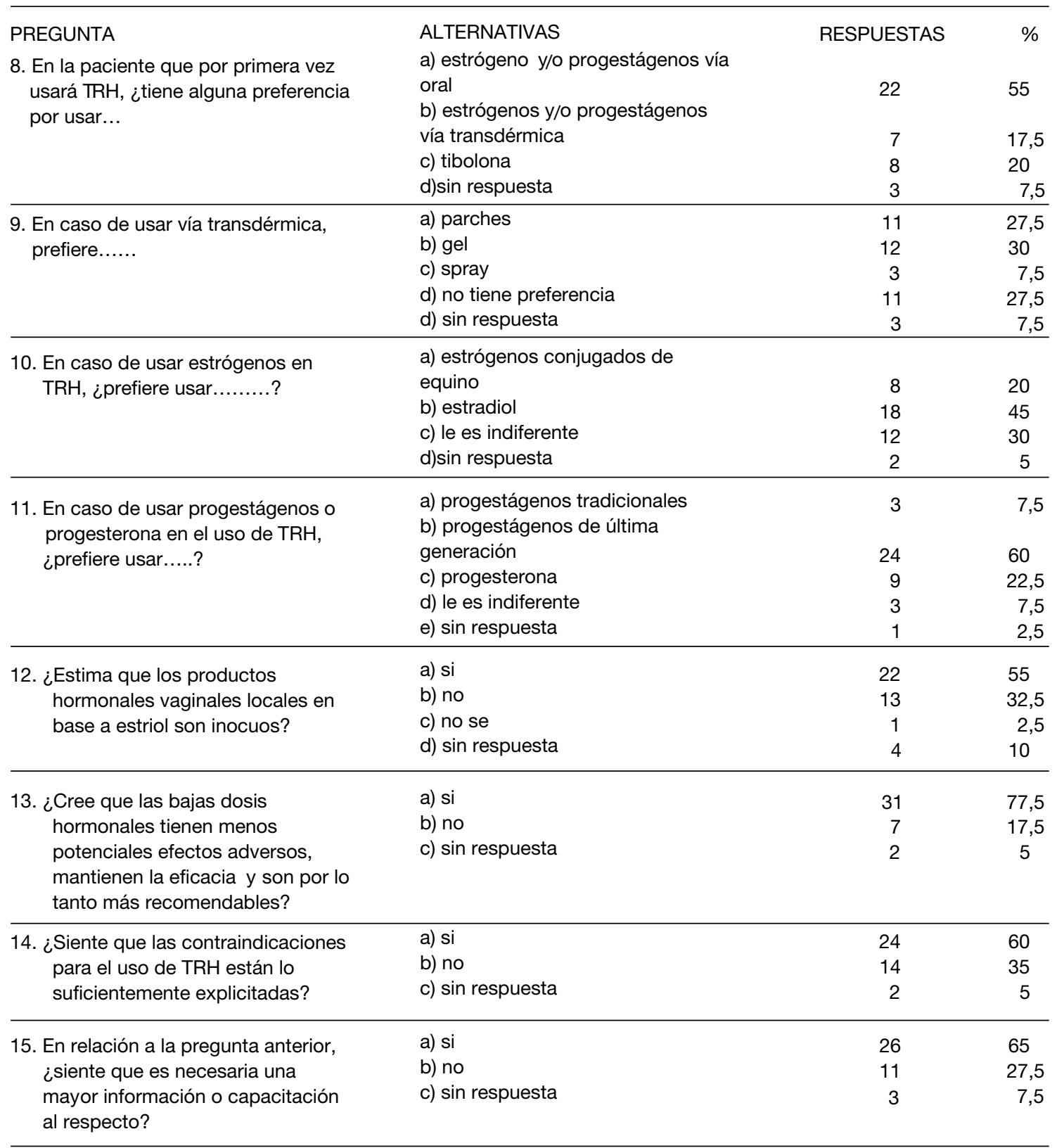

nativas o productos naturales en vez de las $\mathrm{TRH}$ tradicionales y que el $80 \%$ de los encuestados percibe que el deseo de uso por parte de las pacientes de estas terapias alternativas ha ido en aumento. Contrariamente a esta percepción por parte de las pacientes, el $85 \%$ de los profesionales NO prefiere el uso de estas terapias alternativas al uso de las
TRH tradicionales siendo solo un 7,5\% (3 profesionales) los que prefieren el uso de estas terapias al uso de las TRH convencionales. El 92,5\% de los encuestados opina que las terapias naturales o alternativas no están lo suficientemente evaluadas en cuanto a su eficacia y seguridad.

Las necesidades de una medicina holística ha 


\section{Tabla III}

\section{PREGUNTAS RELACIONADAS CON EL FACTOR ETARIO Y USO DE TRH}

\begin{tabular}{|c|c|c|c|}
\hline $\begin{array}{l}\text { PREGUNTA } \\
\text { 16. ¿Cree que la edad de la paciente } \\
\text { es un factor importante para } \\
\text { considerar en la indicación de la } \\
\text { TRH? }\end{array}$ & $\begin{array}{l}\text { ALTERNATIVAS } \\
\text { a) si } \\
\text { b)no } \\
\text { c)sin respuesta }\end{array}$ & $\begin{array}{c}\text { RESPUESTAS } \\
37 \\
2 \\
1\end{array}$ & $\begin{array}{c}\% \\
92,5 \\
5 \\
2,5\end{array}$ \\
\hline $\begin{array}{l}\text { 17. ¿Estima que la TRH en la paciente } \\
\text { asintomática, entre } 50 \text { y } 60 \text { años } \\
\text { y en caso de no existir } \\
\text { contraindicaciones específicas, } \\
\text { debe ser usada idealmente? }\end{array}$ & $\begin{array}{l}\text { a) entodas las pacientes } \\
\text { b) en las que lo soliciten } \\
\text { solamente } \\
\text { c) en ninguna paciente } \\
\text { d) sin respuesta }\end{array}$ & $\begin{array}{r}8 \\
18 \\
10 \\
4\end{array}$ & $\begin{array}{l}20 \\
45 \\
25 \\
10\end{array}$ \\
\hline $\begin{array}{l}\text { 18....en las mismas condiciones de la } \\
\text { pregunta anterior, pero en } \\
\text { pacientes de más de } 60 \text { años, } \\
\text { ¿debiera ser usada...? }\end{array}$ & $\begin{array}{l}\text { a) en todas las pacientes } \\
\text { b) en las que lo soliciten } \\
\text { solamente } \\
\text { c) en ninguna paciente } \\
\text { d) sin respuesta }\end{array}$ & $\begin{array}{r}1 \\
13 \\
23 \\
3\end{array}$ & $\begin{array}{r}2,5 \\
32,5 \\
57,5 \\
7,5\end{array}$ \\
\hline $\begin{array}{l}\text { 19. Y en el grupo de pacientes } \\
\text { sintomáticas de climaterio, sin } \\
\text { contraindicaciones y más de } 60 \\
\text { años ¿la usa usted? }\end{array}$ & $\begin{array}{l}\text { a) entodas las pacientes } \\
\text { b) en las que lo soliciten } \\
\text { solamente } \\
\text { c) en ninguna paciente } \\
\text { d) sin respuesta }\end{array}$ & $\begin{array}{r}8 \\
22 \\
6 \\
4\end{array}$ & $\begin{array}{l}55 \\
15 \\
10\end{array}$ \\
\hline $\begin{array}{l}\text { 20. ¿Considera que hay un límite } \\
\text { superior de edad para indicar el } \\
\text { uso de TRH? }\end{array}$ & $\begin{array}{l}\text { a) si } \\
\text { b) no } \\
\text { c)sinrespuesta }\end{array}$ & $\begin{array}{r}21 \\
18 \\
1\end{array}$ & $\begin{array}{l}52,5 \\
45 \\
2,5\end{array}$ \\
\hline $\begin{array}{l}\text { 21. ¿Cree que hay límites de tiempo } \\
\text { máximo de uso de la TRH? }\end{array}$ & $\begin{array}{l}\text { a) si } \\
\text { b) no } \\
\text { c) sin respuesta }\end{array}$ & $\begin{array}{r}28 \\
11 \\
1\end{array}$ & $\begin{array}{r}70 \\
27,5 \\
2,5\end{array}$ \\
\hline
\end{tabular}

forzado a muchos ginecólogos en el tener que incursionar, de acuerdo a su propio criterio y posibilidad, en el ideal de otorgar una medicina integral a sus pacientes. Al respecto, un $15 \%$ de los encuestados indica fármacos para el tratamiento de la hipertensión arterial, un $45 \%$ para el tratamiento de la hipercolesterolemia y un $87,5 \%$ para la osteopeniaosteoporosis.

Nos pareció también importante evaluar la magnitud de la indicación de TRH en fragmentos de grupos etarios asociados o no a sintomatología climatérica; las distintas formulaciones hormonales a preferir usar en la candidata que por primera vez usará TRH, así como las vías de administración de las mismas. A este respecto, el 55\% de los encuestados prefiere indicar en la paciente que usará por primera vez TRH estrógenos y/o progestágenos por vía oral siendo que en las mismas circunstancias un $17,5 \%$ prefiere el uso de los mismos productos por vía transdérmica, y un $20 \%$ prefiere tibolona, en tanto 3 profesionales no responden esta pregunta $(7,5 \%)$. Podría especularse que la preferencia general por el uso de tibolona podría ser mayor, dado que el consejo para su indicación es cuando la menopausia esta claramente instituida, idealmente con un año o más de amenorrea. También nos pareció importante saber opiniones sobre los costos de los medicamentos y por la preferencia de los encuestados por productos farmacológicos originados en las industrias médicas que efectúan investigación.

Un ínfimo número de preguntas formuladas no son respondidas por los encuestados; así se señala en cada caso de ocurrir esta circunstancia en la sección resultados; lo que nos parece el confirmar el hecho de que por su alto grado de especialización algunos de los ginecólogos de nuestro Departamento han descartado el tratamiento y manejo de este tipo de pacientes.

La observación que en una de las excepcionales preguntas que encierran un conocimiento de un 


\section{Tabla IV \\ PREGUNTAS RELACIONADAS RESPECTO A PERCEPCIONES Y ACTITUDES POS ESTUDIO WHI}

\begin{tabular}{|c|c|c|c|}
\hline PREGUNTA & ALTERNATIVAS & RESPUESTAS & $\%$ \\
\hline 22. ¿Siente que después del Estudio & a) si & 32 & 80 \\
\hline $\begin{array}{l}\text { WHI, ha cambiado su actitud } \\
\text { sobre el uso de TRH? }\end{array}$ & b) no & 8 & 20 \\
\hline \multirow{4}{*}{$\begin{array}{l}\text { 23. A cinco años de la publicación } \\
\text { del Estudio WHI, los datos } \\
\text { aportados por él, le parecen....... }\end{array}$} & a) altamente fiables & 5 & 12,5 \\
\hline & b) medianamente fiables & 24 & 60 \\
\hline & c) no fiables & 2 & 5 \\
\hline & $\begin{array}{l}\text { d) estoy incapacitado para } \\
\text { opinar }\end{array}$ & 9 & 22,5 \\
\hline \multirow{5}{*}{$\begin{array}{l}\text { 24. El hecho que en el estudio WHI } \\
\text { el grupo tratado con estrógenos } \\
\text { conjugados solos, en las } \\
\text { mujeres histerectomizadas, } \\
\text { haya tenido un } 23 \% \text { menos de } \\
\text { cáncer de mama, que el grupo } \\
\text { placebo, después de } 7 \text { años de } \\
\text { uso, le parece ser un hecho..... }\end{array}$} & a) inesperado y no fiable & 9 & 22,5 \\
\hline & b) esperado fiable & 5 & 12,5 \\
\hline & c) esperado no fiable & 1 & 2,5 \\
\hline & d) sin respuesta & 3 & 7,5 \\
\hline & e) no sé & 2 & 5 \\
\hline \multirow{4}{*}{$\begin{array}{l}\text { 25. Basado en los hallazgos del } \\
\text { estudio WHI en la pregunta } \\
\text { anterior, ¿indicaría usted } \\
\text { estrógenos en mujeres } \\
\text { histerectomizadas como TRH, } \\
\text { teniendo éstas factores de } \\
\text { riesgo aumentados de cáncer de } \\
\text { mama? }\end{array}$} & a) si & 14 & 35 \\
\hline & b) no & 22 & 55 \\
\hline & c) no se & 2 & 5 \\
\hline & d) sin respuesta & 2 & 5 \\
\hline
\end{tabular}

26. Desde el punto de vista de un eventual mayor riesgo de cáncer de mama en usuarias de $\mathrm{TRH}$, a) existe una significativa asociación con todo tipo de TRH 10 estima que........

b) existe asociación significativa con algunos tipos y dosis de

$\begin{array}{lll}\mathrm{TRH} \text { solamente } & 35 & 87,5\end{array}$

c) no existe asociación

$\begin{array}{lcc}\text { significativa } & 1 & 2,5\end{array}$

\begin{tabular}{|c|c|c|c|}
\hline 27. ¿Estima que el raloxifeno, es un & a) si & 24 & 60 \\
\hline aporte en la prevención del & b) no & 8 & 20 \\
\hline cáncer de mama en las mujeres & c) no sé & 4 & 10 \\
\hline de más de 50 años? & d) sin respuesta & 4 & 10 \\
\hline
\end{tabular}

\begin{tabular}{llrl}
\hline 28. Su inquietud a ser demandado & a) alto & 6 & 15 \\
en caso de ocurrir un evento & b) mediano & 9 & 22,5 \\
adverso serio con la TRH por & c) bajo & 24 & 60 \\
usted indicada, es....... & d) sin respuesta & 1 & 2,5 \\
\hline
\end{tabular}

tema específico, más que una percepción o actitud médica, sea la que haya tenido por lejos la mayor incidencia de respuestas "no sé", o "sin respuesta" ( $20 \%$ en total, para pregunta estima que "el raloxifeno es un aporte en la prevención del cáncer de mamas en mujeres mayores de 50 años") nos hace pensar en la idoneidad y seriedad con la cual cada uno de los encuestados respondió cada una de las preguntas formuladas.

El análisis comparativo de las respuestas entre los 10 ginecólogos que se autoconsideran especialistas en climaterio y menopausia y los 30 restantes 


\section{Tabla V}

\section{PREGUNTAS RESPECTO AL USO DE MEDICINAS ALTERNATIVAS A LA TRH Y FACTORES RELACIONADOS CON LA DISCONTINUACIÓN DE LA TRH MISMA}

\begin{tabular}{|c|c|c|c|}
\hline $\begin{array}{l}\text { PREGUNTA } \\
\text { 29. Su percepción sobre la confianza y } \\
\text { deseo por parte de las pacientes } \\
\text { al uso de medicinas alternativas y } \\
\text { productos naturales en vez de la } \\
\text { TRH tradicional es.............. }\end{array}$ & $\begin{array}{l}\text { ALTERNATIVAS } \\
\text { a) alto } \\
\text { b) mediano } \\
\text { c) bajo } \\
\text { d) sin respuesta }\end{array}$ & $\begin{array}{l}\text { RESPUESTAS } \\
16 \\
15 \\
8 \\
1\end{array}$ & $\begin{array}{l}\% \\
40 \\
37,5 \\
20 \\
2,5\end{array}$ \\
\hline $\begin{array}{l}\text { 30. ¿Cree que el deseo de su uso, en } \\
\text { el contexto de la pregunta } \\
\text { anterior, ha ido aumentando por } \\
\text { parte de las pacientes? }\end{array}$ & $\begin{array}{l}\text { a) si } \\
\text { b) no } \\
\text { c) sin respuesta }\end{array}$ & $\begin{array}{r}32 \\
7 \\
1\end{array}$ & $\begin{array}{r}80 \\
17,5 \\
2,5\end{array}$ \\
\hline $\begin{array}{l}\text { 31. En estos momentos, ¿prefiere } \\
\text { usted frecuentemente el uso de } \\
\text { medicinas alternativas o } \\
\text { productos naturales al uso de } \\
\text { TRH tradicional? }\end{array}$ & $\begin{array}{l}\text { a) si } \\
\text { b) no } \\
\text { c) sin respuesta }\end{array}$ & $\begin{array}{r}3 \\
34 \\
3\end{array}$ & $\begin{array}{c}7,5 \\
85 \\
7,5\end{array}$ \\
\hline $\begin{array}{l}\text { 32. ¿Cree que los usos de productos } \\
\text { alternativos de origen natural, } \\
\text { están lo suficientemente } \\
\text { evaluados en cuanto a su eficacia } \\
\text { yseguridad? }\end{array}$ & $\begin{array}{l}\text { a) si } \\
\text { b) no }\end{array}$ & $\begin{array}{r}3 \\
37\end{array}$ & $\begin{array}{r}7,5 \\
92,5\end{array}$ \\
\hline
\end{tabular}

33. ¿Cuáles de estos factores cree que tienen importancia trascendencia en la discontinuación de la TRH en su práctica diaria?

a. Inseguridad suya, de los reales beneficios de la TRH
a) si
13
32,5
b) no

b. Médico tratante dubitativo sobre la magnitud real de riesgos
a) si
b) no
c) sin respuesta

18

c. Influencia negativa sobre uso de TRH en la prensa
a) si
b) no
c) sin respuesta

d. Influencia negativa de uso de TRH por médicos de otras especialidades (cardiólogos, oncólogos, etc.)

si
no
sin respuesta

e. Influencia negativa sobre uso de TRH por amistades o parientes de la paciente
a) $\mathrm{si}$
b) no
c) $\sin$ respuesta

f. Influencia de industria

farmacéutica de medicinas de origen natural
a) $\mathrm{si}$
b) no 
que responden desde el punto de vista de un ginecólogo general será presentado en una próxima comunicación.

Esta encuesta nos ha servido para establecer en el momento actual un diagnóstico de situación respecto a las percepciones y actitudes de los médicos miembros de nuestro Departamento con respecto al uso de TRH. El evidenciar que el $35 \%$ de ellos estima que las contraindicaciones para el uso de $\mathrm{TRH}$ no están lo suficientemente explicitadas, con el agregado que el $65 \%$ de los encuestados siente que es necesaria una mayor información y capacitación al respecto, nos motiva y obliga más que a tomar una activa actitud al respecto sino al profundo deber de un selectivo autoperfeccionamiento por parte de cada uno de los médicos miembros de nuestra Unidad de Climaterio y Menopausia respecto a los usos o abusos de la $\mathrm{TRH}$ con la consecuente difusión de la información a los ginecoobstetras generales miembros o no de nuestro Departamento, así como a médicos generales o en etapa de formación. Las Guías y/o Consensos Nacionales o Internacionales han sido y son una fundamental contribución al respecto, como así también los selectivos cursos de perfeccionamiento, como el recientemente realizado en nuestro país por la Sociedad Chilena de Menopausia y Climaterio y la Sociedad Internacional de Menopausia (18).

Tan importante como las conclusiones anteriormente enunciadas es el fomento de la educación de las actuales y potenciales futuras usuarias de $\mathrm{TRH}$, como claramente de este estudio se desprende. Sólo la adecuada capacitación informativa de la comunidad médica y de las usuarias permitirá tomar la decisión más adecuada en una materia médica que aún se desenvuelve en el apasionante y enigmático campo que la medicina por si sola encierra. El participar en el lograr una vida madura, plena y positiva, y el derecho a una longevidad digna, es un deber de todos. Por último, en tanto la información científica sobre esta temática no esté lo suficientemente decantada o establecida, aparece lógico que el manejo de este tipo de pacientes esté a cargo de los grupos médicos altamente especializados en la materia.

Agradecimientos y constancia: A todos los médicos de nuestro Departamento, cada uno de los cuales contribuyó con sus respuestas a la ejecución de este estudio. Este estudio no ha recibido en su realización ningún aporte tanto en lo ideológico como en lo económico de empresa o institución.

\section{BIBLIOGRAFÍA}

1. Writing Group for the Women`s Health Initiative In- vestigators. Risks and benefits of estrogen plus progestin in healthy postmenopausal women. JAMA 2002;288:321-33.

2. FDA. Terapia hormonal a dosis bajas.pdf. Press release, 2004.

3. Jovell A, Navarro-Rubio MD. Evaluación de la evidencia científica. Medicina Clínica 1996;105:740-3.

4. The Women's Health Initiative Steering Committee. Effects of conjugated equine estrogen in postmenopausal women with hysterectomy. JAMA 2004;291:170112.

5. Hsia J, Langer RD, Manson JE, et al. For the Women Health Initiative Investigators. Conjugated equine estrogens and coronary disease: the Women Health Initiative. Arch Intern Med 2006;166:357-65.

6. Sturdee DW, MacLennan A. Editorial: The pendulum swings back; estrogen is now benefical if started at the right time. Climateric 2006;9:73-4.

7. Pines A, Sturdee DW, Birkhauser M. For the International Menopause Society. Press Statement, February 13, 2006. More data on hormone therapy and coronary heart disease; comments on recent publications from the WHI and Nurses Health Study. Climateric 2006;9:75-6.

8. Rossouw JE, Prentice RL, Manson, JE, et al. Postmenopausal hormone therapy and risk of cardiovascular disease by age and years since menopause. JAMA 2007;297:1465-77.

9. Manson JE, Allison M, Rossouw J, et al. Estrogen therapy and coronary-artery calcification. $\mathrm{N}$ Engl J Med 2007;356:2591-602.

10. MacLennan AH, Henderson VW, Paine BJ, et al. Hormone therapy, timing of initiation and cognition in women older than 60 years: the remember pilot study. Menopause 2006;13:28-36.

11. Alexandersen $P$, Tanko LB, Bagger $Y Z$, et al. The long-term impact of 2-3 years of hormone replacement therapy on cardiovascular mortality and atherosclerosis in healthy women. Climateric 2006;9:108-18.

12. Grodstein F, Manson JE, Stampfer MJ. Hormone therapy and coronary heart disease: the role of time since menopause and age hormone initiation. J Women Health 2006;15:35-44.

13. Phillips LS, Langer RD. Posmenopausal hormone therapy: critical reappraisal and a unified hypothesis. Fertil Steril 2005;83:558-66.

14. Salpeter SR. Hormone therapy for younger postmenopausal women: how can we make sense out of the evidence? Climateric 2005;8:307-10.

15. Hersh AL, Stefanick ML, Stafford RS. National use of postmenopausal hormone therapy: annual trends and response to recent evidence. JAMA 2004;291:47-53.

16. Basado en datos IMS sales, 2006. Hallado en: www. imshealth.com.

17. Basado en datos IMS sales, Q4, 2005. Hallado en: www.imshealth.com.

18. The International Menopause Society and The Chilean 
Board School on the Menopause. Organizado por: Sociedad Chilena del Climaterio and the International Menopause Society. Chairman: Dr. Luis Cruzat, Prof.
Amos Pines, Co-Charmain: Dr. Sergio Brantes, Dr. David W. Sturdee. 3-4 Agosto 2007, Santiago, Chile. 